\title{
THE RAVE CATALOG OF STELLAR ELEMENTAL ABUNDANCES: FIRST DATA RELEASE
}

\author{
C. Boeche $^{1}$, A. Siebert ${ }^{2}$, M. Williams ${ }^{1}$, R. S. De Jong ${ }^{1}$, M. Steinmetz ${ }^{1}$, J. P. Fulbright ${ }^{3}$, G. R. Ruchti ${ }^{3}$, \\ O. Bienaymé ${ }^{2}$, J. Bland-Hawthorn ${ }^{4}$, R. Campbell ${ }^{5}$, K. C. Freeman ${ }^{6}$, B. K. Gibson ${ }^{7}$, G. Gilmore $^{8}$, \\ E. K. Grebel ${ }^{9}$, A. Helmi ${ }^{10}$, U. Munari ${ }^{11}$, J. F. Navarro ${ }^{12}$, Q. A. Parker ${ }^{13}, 14$, W. Reid ${ }^{13}$, G. M. Seabroke ${ }^{15}$,

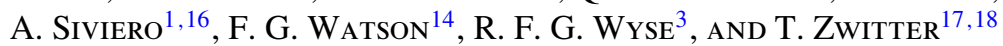 \\ ${ }^{1}$ Leibniz-Institut für Astrophysik Potsdam (AIP), D-14482 Potsdam, Germany \\ ${ }^{2}$ Observatoire Astronomique de Strasbourg, Université de Strasbourg, CNRS, UMR 7550, F-67000 Strasbourg, France \\ ${ }^{3}$ Department of Physics and Astronomy, Johns Hopkins University, Baltimore, MD 21218, USA \\ ${ }^{4}$ Sydney Institute for Astronomy, School of Physics A28, University of Sydney, NSW 2006, Australia \\ 5 Department of Physics and Astronomy, Western Kentucky University, Bowling Green, KY, USA \\ ${ }^{6}$ Research School of Astronomy and Astrophysics, Australia National University, Weston Creek, Canberra ACT 2611, Australia \\ 7 Jeremiah Horrocks Institute, University of Central Lancashire, Preston PR1 2HE, UK \\ ${ }^{8}$ Institute of Astronomy, University of Cambridge, Madingley Road, Cambridge CB3 OHA, UK \\ ${ }^{9}$ Astronomisches Rechen-Institut, Zentrum für Astronomie der Universität Heidelberg, D-69120 Heidelberg, Germany \\ ${ }^{10}$ Kapteyn Astronomical Institute, University of Groningen, 9700 AV Groningen, The Netherlands \\ ${ }^{11}$ INAF Osservatorio Astronomico di Padova, Asiago I-36012, Italy \\ 12 Department of Physics and Astronomy, University of Victoria, Victoria BC V8W 3P6, Canada \\ ${ }^{13}$ Department of Physics and Astronomy, Faculty of Sciences, Macquarie University, Sydney, NSW 2109, Australia \\ ${ }^{14}$ Australian Astronomical Observatory, Epping, NSW 1710, Australia \\ ${ }^{15}$ Mullard Space Science Laboratory, University College London, Holmbury, St. Mary RH5 6NT, UK \\ ${ }^{16}$ Dipartimento di Astronomia, Universitá di Padova, I-35122 Padova, Italy \\ ${ }^{17}$ Faculty of Mathematics and Physics, University of Ljubljana, 1000 Ljubljana, Slovenia \\ ${ }^{18}$ Center of Excellence SPACE-SI, 1000 Ljubljana, Slovenia \\ Received 2011 June 1; accepted 2011 September 20; published 2011 November 11
}

\begin{abstract}
We present chemical elemental abundances for 36,561 stars observed by the RAdial Velocity Experiment (RAVE), an ambitious spectroscopic survey of our Galaxy at Galactic latitudes $|b|>25^{\circ}$ and with magnitudes in the range $9<I_{\text {DENIS }}<13$. RAVE spectra cover the Ca-triplet region at 8410-8795 $\AA$ with resolving power $R \sim 7500$. This first data release of the RAVE chemical catalog is complementary to the third RAVE data release of radial velocities and stellar parameters, and it contains chemical abundances for the elements $\mathrm{Mg}, \mathrm{Al}, \mathrm{Si}, \mathrm{Ca}, \mathrm{Ti}, \mathrm{Fe}$, and $\mathrm{Ni}$, with a mean error of $\sim 0.2$ dex, as judged from accuracy tests performed on synthetic and real spectra. Abundances are estimated through a dedicated processing pipeline in which the curve of growth of individual lines is obtained from a library of absorption line equivalent widths to construct a model spectrum that is then matched to the observed spectrum via a $\chi^{2}$ minimization technique. We plan to extend this pipeline to include estimates for other elements, such as oxygen and sulfur, in future data releases.
\end{abstract}

Key words: catalogs - Galaxy: abundances - Galaxy: evolution - stars: abundances - surveys - techniques: spectroscopic

Online-only material: machine-readable and VO tables

\section{INTRODUCTION}

Stars inherit the chemical patterns of the interstellar matter from which they were born. At the end of their lives they return their nuclear products to the interstellar medium through stellar winds and supernovae, enriching it with heavier elements. The elemental abundance pattern of every generation of stars thus depends on the previous one and, in time, a sort of "genealogical tree" develops. In principle, this allows for the star formation history of a galaxy to be traced using stellar elemental abundances (Freeman \& Bland-Hawthorn 2002).

At the same time, the assembly history of a galaxy also leaves traces in the kinematics of its stars. Indeed, once born, stars behave like a collisionless fluid that spreads through phase space, generally leaving clear tracks of their dynamical origin. For example, disrupted open clusters generate moving groups of stars with similar kinematics that can still be recognized long after disruption (Eggen 1965), while accretion events produce transient stellar streams that closely track the orbit of their progenitor satellites (Helmi et al. 2006).
Chemical and kinematic information, when available, can therefore be used to reconstruct the history of the Milky Way much in the way archaeologists examine relics to recreate the ancient past. The data requirements of this exercise, however, have limited its applicability in the past: assembling a large and homogeneous set of proper motions, distances, radial velocities, and elemental abundances requires large photometric and spectroscopic surveys that have not been feasible until recently.

Spectroscopy has traditionally been the bottleneck, with much of the work on chemical elemental abundances limited to small, biased samples. Until recently, iron abundances $([\mathrm{Fe} / \mathrm{H}])$ had been estimated for samples as large as the $\sim 16,000$ stars of the Geneva-Copenhagen Survey (Nordström et al. 2004), but the numbers of stars with abundances measured for multiple elements were much smaller. Indeed, the largest homogeneous sample available until now was published by Valenti \& Fisher (2005), who measured the abundances of five elements in 1040 nearby F, G, and K stars.

Smaller samples, often composed of a few hundred stars or fewer, have also been presented by several authors (Edvardsson 
et al. 1993; Fuhrmann 1998, 2008; Luck \& Heiter 2006, 2007; Reddy et al. 2006, among others). Larger, but inhomogeneous, data sets have been collated from the literature by Soubiran \& Girard (2005) and Venn et al. (2004), who compiled 743 and 821 stars, respectively, and also in the PASTEL catalog (Soubiran et al. 2010), which is a collection of 865 literature studies.

The availability of dedicated telescopes and multi-object spectrographs has radically changed this state of affairs, enabling surveys such as the RAdial Velocity Experiment (RAVE; Steinmetz et al. 2006), and SEGUE (Yanny et al. 2009). Combined, these two surveys have now taken spectra for roughly a million stars. Galactic archaeology has thus become one of the fastest-growing fields of astronomical enquiry, as evidenced by the numerous surveys currently underway or in the advanced planning stages, e.g., Gaia (Perryman et al. 2001), LAMOST $^{19}$, and HERMES (Freeman \& Bland-Hawthorn 2008).

We present here the first release of the chemical catalog for RAVE, an ambitious spectroscopic survey of our Galaxy at Galactic latitudes $|b|>25^{\circ}$ and magnitudes in the range $9<I_{\text {DENIS }}<13$. This catalog contains multi-element abundance measurements for 36,561 stars of the Milky Way based on 37,848 RAVE spectra covering the Ca-triplet region at 8410-8795 $\AA$ with resolving power $R \sim 7500$. This data release is associated with the RAVE third data release (DR3; Siebert et al. 2011), where further information (kinematics, photometry, etc.) can be found. As of summer 2011, RAVE has taken more than half a million spectra for some 400,000 stars. Abundances for these targets will be published in subsequent data releases.

RAVE (Steinmetz et al. 2006) was first conceived as a radial velocity survey to provide the missing third velocity component for stars in the solar suburb. However, it soon became clear that RAVE spectra carry much more information than just radial velocities. After further development, the RAVE processing pipeline was modified to deliver estimations of the values of stellar parameters like effective temperature, gravity, and metallicity (Zwitter et al. 2008; Siebert et al. 2011).

We take this development one step further here by tackling the measurement of chemical elemental abundances at the medium spectral resolution provided by RAVE data. This feature is shared with other large spectroscopic surveys with limited resolution and spectral coverage, such as Gaia and LAMOST. Although high precision is not expected from medium resolution spectroscopy, the availability of hundreds of thousands of RAVE spectra enables the creation of a large and homogeneous catalog of chemical abundances suitable for statistical investigation.

The RAVE chemical pipeline measures abundances for seven elements: $\mathrm{Mg}, \mathrm{Al}, \mathrm{Si}, \mathrm{Ca}, \mathrm{Ti}, \mathrm{Fe}$, and $\mathrm{Ni}$. Homogeneity is assured by an automated processing pipeline that measures abundances assuming the stellar parameters computed by the RAVE pipeline described in Siebert et al. (2011). RAVE stars have astrometry from different sources like Tycho2 (Høg et al. 2000), PPMX (Roeser et al. 2008), and UCAC2 (Zacharias et al. 2004). These, together with RAVE radial velocities and distance estimates (Breddels et al. 2010; Zwitter et al. 2010; Burnett et al. 2011), yield three-dimensional positions and velocities. Combining this information with chemical abundances results in a unique chemo-kinematic data set suitable for investigations of the formation history of the Galaxy.

This paper is structured as follows. In Section 2, we present the chemical processing pipeline, detailing how the chemical

\footnotetext{
19 http://www.lamost.org/website/en
}

elemental abundances are measured. Tests to establish the accuracy and reliability of the results are detailed in Section 3, while in Section 4 we discuss our method of measurement, and in Section 5 we present the RAVE chemical catalog. In Section 6 we outline our conclusions.

\section{THE PIPELINE}

The RAVE chemical abundance pipeline uses a different approach to classical elemental abundance estimation methods. These methods either (1) measure equivalent widths (EW)s and infer elemental abundances from the curves-of-growth (COGs) or (2) synthesize spectra with varying elemental abundances to find the best match between the synthetic and observed spectra. The first method cannot be successfully applied to the RAVE spectra because it requires isolated lines for precise EW measurements and, at the medium resolution of RAVE, most of the lines are instrumentally blended. Also, the second method is computationally too expensive as it requires the synthesis of several spectra $\sim 400 \AA$ wide, each having hundreds of lines.

The adopted method can be considered a hybrid approach, measuring the elemental abundances by fitting the spectrum with a model constructed with lines of known EWs. The construction of the model is robust and fast, with the elemental abundances determined by a $\chi^{2}$ minimization routine. The drawback of this method is that the EWs of the lines are computed by neglecting the opacity of the neighboring lines. This may overestimate the EWs, leading in some cases to underestimated elemental abundances. We show below that this effect is small for most of the lines in the RAVE wavelength range.

\subsection{Procedure}

The chemical pipeline utilizes RAVE reduced spectra and their stellar parameters, such as effective temperature $\left(T_{\mathrm{eff}}^{\mathrm{RAVE}}\right)$, gravity $\left(\log g^{\mathrm{RAVE}}\right)$, and metallicity $\left([\mathrm{m} / \mathrm{H}]^{\mathrm{RAVE}}\right)$, returned by the RAVE pipeline (described in Siebert et al. 2011). ${ }^{20}$ The pipeline also uses an EW library based on the RAVE line list. The RAVE line list contains 604 known absorption lines identified in the RAVE wavelength range (Section 2.2). The EW library contains the EWs of absorption lines for every point on the $T_{\text {eff }}, \log g$, and $[\mathrm{m} / \mathrm{H}]$ grid (Section 2.3), which accounts for the variation of the EW with the stellar parameters. Variations of EW with elemental abundances are then further calculated for each point of this grid, for five different abundance levels in the range $[-0.4,0.4]$ with respect to the value of metallicity $[\mathrm{m} / \mathrm{H}]$.

The pipeline contains some auxiliary codes to (re)normalize the spectrum and detect spectral defects like bad normalization, cosmic rays, and other unwanted features.

The pipeline algorithm can be outlined as follows.

1. Upload the normalized, RV corrected, and wavelength calibrated spectrum and the estimated stellar parameters $T_{\mathrm{eff}}^{\mathrm{RAVE}}, \log g^{\mathrm{RAVE}},[\mathrm{m} / \mathrm{H}]^{\mathrm{RAVE}}$.

2. Upload the RAVE line list from the EW library and the corresponding EWs for the stellar parameters at the five different abundance levels.

3. Extract a shorter line list of those lines which, at the estimated stellar parameters, have large enough EWs to be visible above the noise.

4. Fit the strong lines and correct the continuum. ${ }^{20}[\mathrm{~m} / \mathrm{H}]^{\text {RAVE }}$ indicates the uncalibrated metallicity as defined in Zwitter et al.
(2008). 
5. Construct the COGs of the lines by fitting a polynomial function through the five $\mathrm{EW}$ abundance points.

6. Create the model by assuming a Gaussian profile for each line and summing these profiles together.

7. Vary the chemical elemental abundances to obtain different models by changing the EWs of the lines according to their COG.

8. Minimize the $\chi^{2}$ between the models and the observed spectrum to find the best-matching model.

In the following we give further details pertaining to these steps.

\subsection{Line List and Constraining the Oscillator Strength}

The RAVE line list contains 604 absorption lines identified in spectra of the Sun and Arcturus. The lines correspond to the element species N I, O I, Mg I, Al I, Si I, S I, Ca I, Ti I, Ti II, Cr I, $\mathrm{Fe}$ I, Fe II, Co I, Ni I, Zri, and to the CN molecule. In order to get precise chemical elemental abundances from the EWs, we first need reliable atomic parameters for the lines. A critical parameter is the oscillator strength ( $g f$, often expressed as logarithm $\log g f$ ). If precise laboratory measurements are missing for the $\log g f$ values then they are obtained through an inverse spectral analysis to obtain "astrophysical $\log g f$ s." This is the case for the RAVE wavelength region, where the Vienna Atomic Line Database (VALD; Kupka et al. 1999) reports that only 11 $\mathrm{Fe}$ I lines have laboratory oscillator strength measurements. The Sun's and Arcturus's spectra were synthesized and the $\log g f$ values for each line were obtained using an automated procedure which varies the $\log g f$ s so as to match both spectra simultaneously. For the synthesis we adopted solar abundances as given by Grevesse \& Sauval (1998). They will be the zero point of the abundances of this work. The details of the procedure are outlined in Appendix A.

The pipeline makes use of a shorter working line list which contains the lines strong enough to be visible above the noise. The lines deemed visible are those satisfying

$$
\mathrm{EW}(\mathrm{m \AA})>2 \cdot \frac{1}{\mathrm{STN}} \cdot d \cdot 1000,
$$

where STN is the signal-to-noise ratio ${ }^{21}$ and $d$ is the spectral dispersion (for RAVE spectra $d=0.4 \AA$ pixel $^{-1}$ ). Other lines can blend with these visible lines, contributing to their flux absorption and changing their apparent EW. We therefore added to the working line list all those lines having EW $>1 \mathrm{~m} \AA$ and being closer than $0.6 \AA$ ( $1.2 \AA$ is the FWHM of a typical RAVE spectrum) to the visible lines.

\subsection{The EW Library and COG Computation}

In order to compute the line COGs we built an EW library with the RAVE line list for 30, 640 atmosphere models. The EWs were computed using the LTE line-analysis software MOOG (Sneden 1973) and the ATLAS9 model atmosphere grid (Castelli \& Kurucz 2003). As the spacing of the ATLAS9 grid is different to our requirements, we linearly interpolated the ATLAS9 grid to produce our own grid, covering the $T_{\text {eff }}$ range $[3600,7600] \mathrm{K}$ in steps of $100 \mathrm{~K}$, gravity range $[0.0,5.0]$ dex in steps of $0.2 \mathrm{dex}$, and metallicities in the range $[-2.5,+0.5]$ dex in steps of 0.1 dex. We did not compute the EWs for atmospheres with $T_{\text {eff }}>5100 \mathrm{~K}$

\footnotetext{
21 In the following, we will use STN to indicate the signal-to-noise ratio as computed in Siebert et al. (2011) whereas $\mathrm{S} / \mathrm{N}$ will be used with the usual meaning given in spectrophotometry.
}

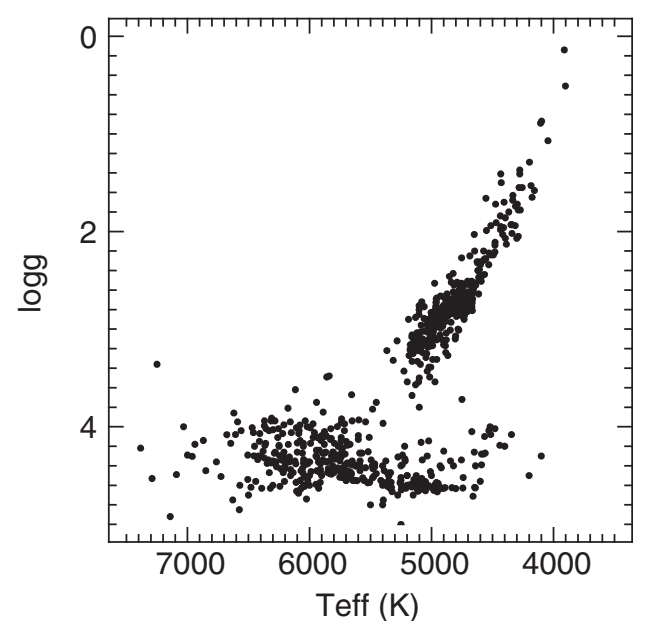

Figure 1. Distribution on $\left(T_{\mathrm{eff}}, \log g\right.$ ) plane of 712 stars observed spectroscopically at high resolution.

and $\log g<1.0$ (hot supergiants), because MOOG does not produce results for these parameters and furthermore there are no such stars in our catalog. For every atmosphere model we computed the EWs of the lines for five abundance levels with respect to iron: $[\mathrm{X} / \mathrm{Fe}]=-0.4,-0.2,0.0,0.2,0.4$ dex assuming $[\mathrm{Fe} / \mathrm{H}]=[\mathrm{m} / \mathrm{H}]$. The whole EW library consists of 145,080 files. To obtain EWs for stellar parameters between the grid points of the EW library we linearly interpolate the closest points on the grid.

\subsection{Microturbulence}

The EW computation requires a value of the microturbulence $\xi$ for each atmosphere model. For high-resolution data $\xi$ is typically determined by measuring the EWs of Fe I lines and changing $\xi$ until the iron abundances inferred from strong and weak lines agree. As we do not measure individual EWs for our spectra we cannot use this procedure, but instead rely on relations giving $\xi$ as a function of the stellar parameters. Such relations have been derived by Edvardsson et al. (1993), Reddy et al. (2003), and Allende Prieto et al. (2004), where $\xi$ is given as a function of $T_{\text {eff }}$ and $\log g$. Unfortunately, these results cover only specific regions of parameter space (e.g., hot dwarfs or cold giants). Thus, we derived our own relation covering a wide a range of $T_{\text {eff }}$ and $\log g$. To do so we made use of literature results from high-resolution spectroscopy that report both stellar parameters and $\xi$ of their stellar samples. We collected data for 712 giants and dwarfs spanning a wide range in $T_{\text {eff }}$ and $\log g$ from Luck \& Heiter (2006, 2007), Bensby et al. (2005), Fuhrmann (1998), Fulbright et al. (2006), and Allende Prieto et al. (2004). Figure 1 displays the coverage in the $T_{\text {eff }}-\log g$ plane for the sample. A third-degree polynomial fit was used to obtain the microturbulence dependence on gravity and effective temperature. Appendix B gives the coefficients of this polynomial fit, $\xi_{\text {poly }}$.

Figure 2 compares the Allende Prieto et al. (2004) law with $\xi_{\text {poly }}$. Uncertainties of $\sigma_{\xi}=0.32 \mathrm{~km} \mathrm{~s}^{-1}$ in our polynomial law translate approximately into $\sim 0.04$ dex elemental abundance uncertainties for dwarfs stars (as estimated by Reddy et al. 2003 and Mishenina et al. 2004).

\subsection{Continuum Re-normalization and Strong Line Fitting}

Before measuring EWs, the spectrum's continuum needs to be re-normalized and its strong lines fitted. While the spectrum 


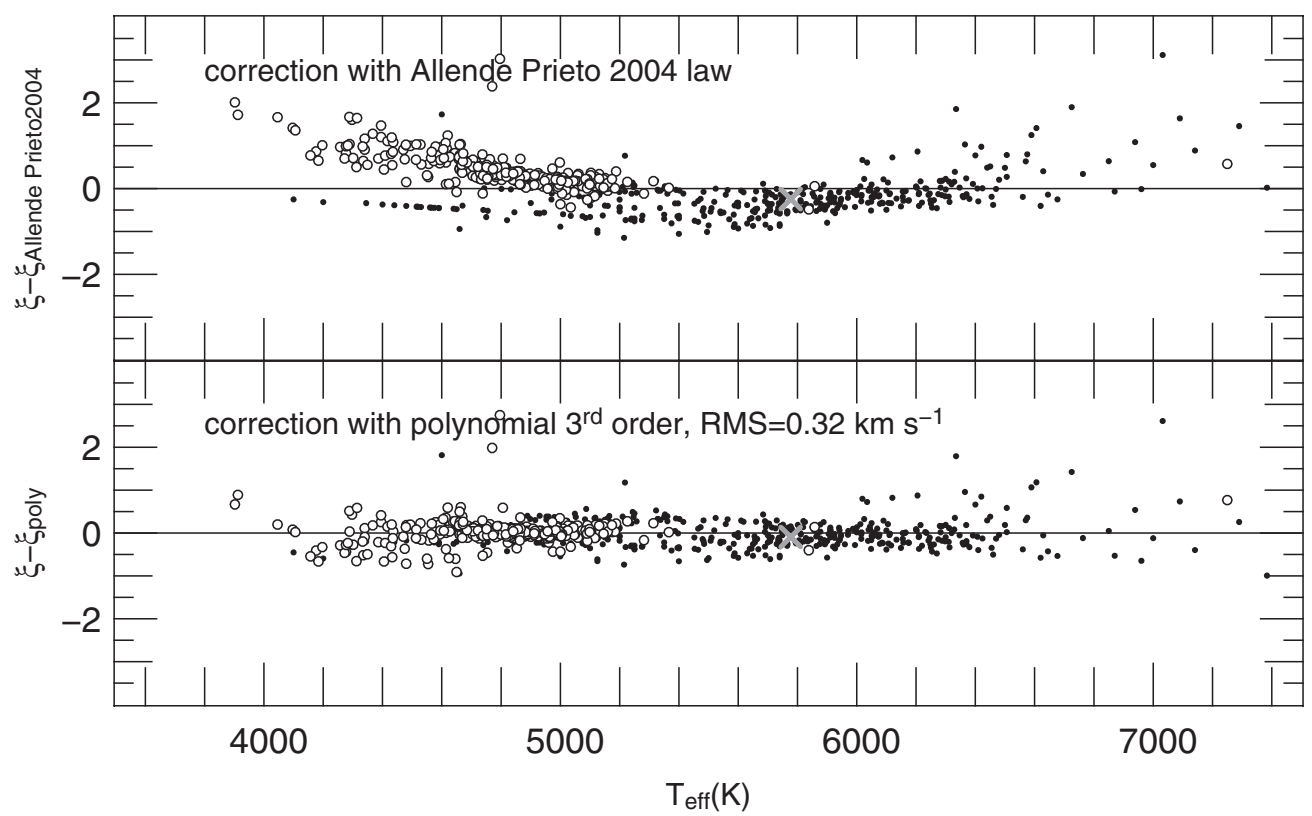

Figure 2. Top panel: residuals between measured microturbulence $\xi$ and computed $\xi$ with the Allende Prieto et al.'s formula for 712 stars. Bottom panel: residuals between measured $\xi$ and computed by a third-degree polynomial function. Solid and open points represent dwarf and giant stars, respectively. The gray cross represents the Sun.

is continuum normalized by the RAVE pipeline, we have found that this normalization is not rigorous enough for elemental abundance estimation: the RAVE pipeline employs a third-order spline function to fit the continuum, leaving behind fringing effects on scales of less than $50 \AA$ wide seen in some RAVEnormalized spectra. The chemical pipeline performs a new normalization to remove such unwanted features, fitting the continuum and the strong $\mathrm{Ca}$ II and $\mathrm{H}$ I Paschen lines. These strong lines are then excluded from the measurement process because they are difficult to fit properly. The pipeline then considers as "continuum" the fit to the classical continuum plus the strong lines, and by comparison with this level the metallic lines are measured.

Before applying the continuum correction we estimate a preliminary metallicity, which we call $[\mathrm{m} / \mathrm{H}]^{\text {est }}$ to distinguish it from the final metallicity $[\mathrm{m} / \mathrm{H}]^{\text {chem }}$. This preliminary metallicity is required because the intensity of the absorption lines must be known in order to subtract them. When they are subtracted the continuum level can then be seen. However, in order to measure the intensity of a line one must know where the continuum lies. The continuum level is therefore determined iteratively, starting with the normalization of the RAVE pipeline and measuring the line intensities using the chemical elemental abundances estimation subroutine (see Section 2.6), with the difference that all the abundances vary together as one variable $[\mathrm{X} / \mathrm{H}]=[\mathrm{m} / \mathrm{H}]^{\text {est }}$. Wavelength intervals $\sim 20 \AA$ wide centered on the strong lines are avoided as the large wings of these lines can affect the results of the $\chi^{2}$ determination of the metallicity. The best-match $[\mathrm{m} / \mathrm{H}]^{\text {est }}$ is then used to synthesize the metallic lines, which are then subtracted before the strong lines are fit.

Fitting the continuum is performed in four steps, summarized below and illustrated in Figure 3 from top to bottom.

1. Perform a preliminary metallicity and subtract the metallic lines (gray line in panel (a), Figure 3).

2. Fit the strong lines with a Lorentzian profile for $\mathrm{Ca}$ II and a Gaussian profile for $\mathrm{H}$ I and subtract them (gray line in panel (b), Figure 3).

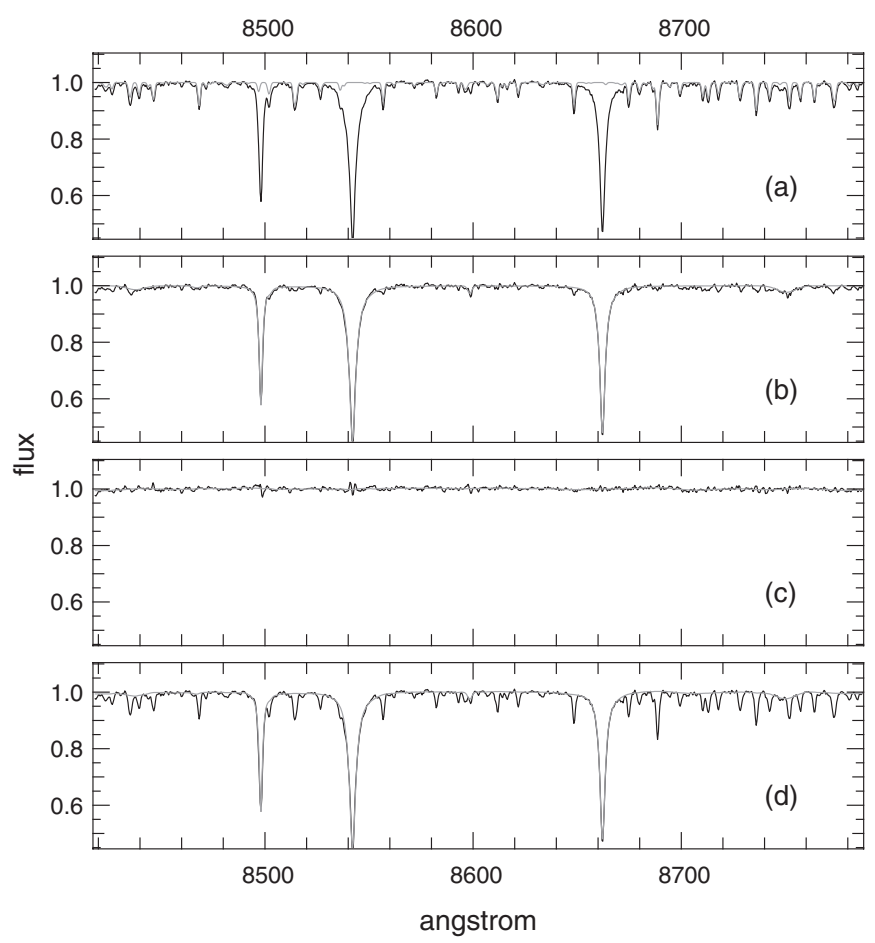

Figure 3. Black line: RAVE spectrum of the Sun. (a) Metallic lines fitting. (b) Strong line fitting. (c) Continuum fitting. (d) The gray line is the sum of the gray lines in panels (b) and (c). This curve is used as the continuum line from which we estimate the chemical elemental abundances.

3. Estimate the continuum profile by box-car smoothing what remains (gray line in panel (c), Figure 3).

4. Add the strong lines and the continuum together to obtain the new "continuum" (gray line in panel (d), Figure 3).

\subsection{Chemical Elemental Abundance Estimation}

The chemical pipeline uses the parameters $T_{\mathrm{eff}}^{\mathrm{RAVE}}$ and $\log g^{\mathrm{RAVE}}$ for the elemental abundance estimation. The observed 


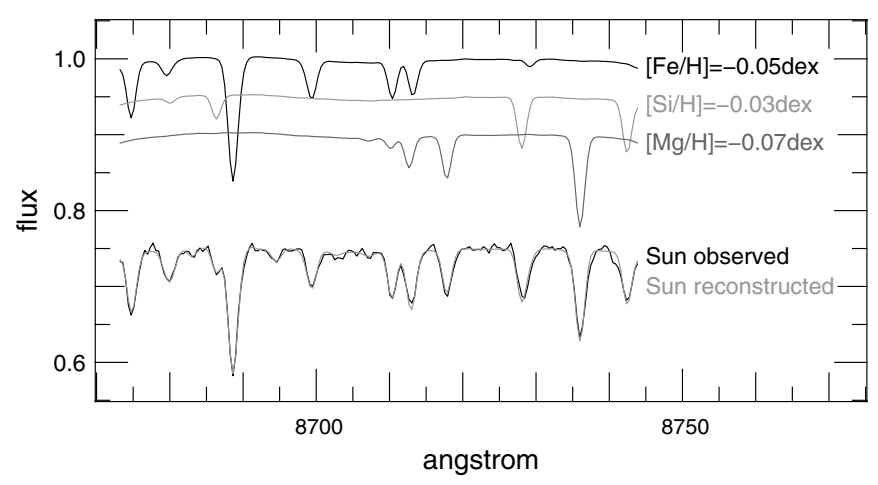

Figure 4. Once the abundances of $\mathrm{Fe}, \mathrm{Si}$, and $\mathrm{Mg}$ are fixed their absorption lines can be synthesized (gray lines on the top). By summing them together we obtain the gray line on the bottom, which is the model spectrum of the Sun. The black line is the Sun's spectrum reflected by the Moon and observed by RAVE.

spectrum is fit by a model spectrum first obtained by subtracting the flux absorbed by the metallic lines to that derived above in Section 2.5, i.e., the strong lines + continuum fit (gray line in panel (d), Figure 3). The absorption lines are assumed to have Gaussian profiles, and as the instrumental profile is dominant with respect to the line profile, we use the same FWHM for all lines. However, this FWHM is varied and optimized during the $\chi^{2}$ minimization process.

The pipeline computes the COG of the lines by using the EWs at five levels of abundances $([\mathrm{X} / \mathrm{Fe}]=-0.4,-0.2,0.0,0.2$, 0.4 dex, assuming $[\mathrm{Fe} / \mathrm{H}]=[\mathrm{m} / \mathrm{H}]^{\mathrm{RAVE}}$ ) from the EW library. For every line, the five EW points are fit with a third-order polynomial function, which serves as the COG. However, these polynomial functions represent only part of the COGs and will diverge if extrapolated too far beyond the five levels in the EW library. To avoid such divergences we limit accepted abundance results to those in the range $-0.6 \leqslant[\mathrm{X} / \mathrm{Fe}] \leqslant+0.6 .^{22}$

Using the COGs the pipeline then creates the model spectrum, using $[\mathrm{X} / \mathrm{H}]=[\mathrm{m} / \mathrm{H}]^{\mathrm{RAVE}}$ as a first guess for the metallicity. It computes the $\chi^{2}$ between the observed spectrum and the model and, through a minimization process, changes the elemental abundances $[\mathrm{X} / \mathrm{H}]$ until the best match (minimum $\chi^{2}$ ) with the observed spectrum is reached. The minimization process is performed with 15 variables: 13 elemental abundances, one molecule, and the instrumental FWHM of the lines.

Figure 4 shows the best-match model spectrum of the Sun and compares it with the observed one. The three spectra on the top show how the model spectrum is built up: the spectra of three elements $(\mathrm{Fe}, \mathrm{Si}, \mathrm{Mg})$ at parameters $T_{\text {eff }}=5861 \mathrm{~K}$ and $\log g=4.54$ are generated according to the estimated elemental abundances and added together to construct the solar spectrum.

This methodology has a drawback: the computed COG of any absorption line neglects the opacity of the neighboring lines, leading to an overestimation of the EW of blended lines and so an underestimation of the elemental abundances. This systematic originates from the EW library created by using the MOOG's driver "ewfind," which computes the expected EW of the lines as if they were isolated. In order to minimize this systematic error we apply a correction coefficient to reduce the EWs of lines which are physically blended. The corrected EW is given by

$$
\mathrm{EW}_{\text {corr }}=\mathrm{EW} \cdot \operatorname{coeff} \cdot \mathrm{cont}
$$

\footnotetext{
22 Abundances beyond this limit can be due to photon noise stronger than the absorption lines or to peculiar abundances. The two cases cannot be distinguished by the chemical pipeline.
}

where the coefficient

$$
\text { coeff }=1-\sum_{i}^{\text {neighbor }<0.2 \AA} \mathrm{EW}_{i} / 2.50 / \text { dispersion }
$$

is computed by considering all the neighboring lines within $0.2 \AA$ of the line of interest. The dispersion is expressed in $\AA$ pixel $^{-1}$ and cont is the value of the continuum (gray line in panel (d) of Figure 3) at the central wavelength of the line. The multiplication of the EW by cont in Equation (1) corrects for the effects of strong lines (such as Ca II) if the line of interest lies within their large wings. This correction reduces the systematic error in the abundances from $\sim-0.15$ dex to $\sim-0.1$ dex or less for most elements (see details in the following sections, where quality checks are outlined).

\subsection{Spectrum Quality Determination: The frac Parameter}

Roughly $25 \%$ of RAVE spectra are affected by defects like fringing or cosmic rays that cannot be removed by continuum correction (they usually affect regions smaller than $100 \AA$ ). In order to determine the locus and size of the defect we define the flux residual between the observed and the best-match model for the $i$ th pixel with

$$
r(i)=f_{\text {model }}(i)-f_{\text {obs }}(i),
$$

where $f$ is the flux of the spectra, and we use the following algorithm (also used in Siebert et al. 2011).

1. Consider the interval $I_{j}=[j-10, j+10]$ centered on the pixel $j$. Compute

$$
\begin{gathered}
\tilde{\chi}^{2}(j)=\frac{1}{\max \left(I_{j}\right)-\min \left(I_{j}\right)} \cdot \sum_{i=\min \left(I_{j}\right)}^{\max \left(I_{j}\right)}\left(\frac{r(i)}{\sigma}\right)^{2}, \\
\psi(j)=\frac{1}{\max \left(I_{j}\right)-\min \left(I_{j}\right)} \sum_{i=\min \left(I_{j}\right)}^{\max \left(I_{j}\right)} r(i),
\end{gathered}
$$

where $\tilde{\chi}^{2}(j)$ is the reduced $\chi^{2}$ and $\psi(j)$ is the estimation of the area between the observed and model spectra. $\sigma$ is the inverse of the signal-to-noise (STN) ratio.

2. If $\tilde{\chi}^{2}(j)>2$ and $\psi(j)>2 \cdot \sigma$ then the pixels in the interval $I_{J}$ are labeled as a defect.

The process is repeated for all the pixels of the spectrum, resulting in an array of 0 and 1, with 1 label indicating nondefective pixels. The fraction of pixels that are non-defective is then denoted with the frac parameter (i.e., the higher the frac parameter, the better the quality of the spectrum). We deem spectra with $f r a c<0.7$ as overly affected by fringing/cosmic ray defects and exclude these spectra from our analysis.

\section{VALIDATION AND ACCURACY}

There are several sources of error that affect the elemental abundances measurement process. For example, there are uncertainties in (1) the oscillator strength values, (2) the continuum normalization, (3) the stellar parameters, (4) microturbulence, (5) uncertainties due to local line opacity neglected by the pipeline, (6) neglect of non-LTE effects, and (7) photon shot noise. These sources of errors interact in a complex fashion, making the error estimation process quite challenging. To establish the capability of the pipeline to derive the 